\title{
ISON NETWORK TRACKING OF SPACE DEBRIS: CURRENT STATUS AND ACHIEVEMENTS
}

\author{
I. Molotov ${ }^{1}$, M. Zakhvatkin ${ }^{1}$, L. Elenin ${ }^{1}$, L. Canals Ros ${ }^{2}$, F. Graziani ${ }^{3}$, P. Teofilatto ${ }^{4}$, T. Schildknecht ${ }^{5}$, \\ S. Ehgamberdiev 6 , A. Aliev ${ }^{6}$, Y. Ivashchenko ${ }^{7}$, R. Zalles ${ }^{8}$, A. Streltsov ${ }^{1}$, A. Krylov ${ }^{1}$, A. Erofeeva ${ }^{1}$, \\ E. Chornaya ${ }^{1}$, O. Rusakov ${ }^{1}$, V. Stepanyants ${ }^{1}$, V. Agletdinov ${ }^{1}$, G. Borovin ${ }^{1}$, and E. Pavlova ${ }^{1}$
}

\begin{abstract}
Interagency International Scientific Optical Network (ISON) represents one of largest systems specializing in observation of space objects. ISON provides permanent monitoring of the whole GEO region, regular surveying of Molniya type orbits, and tracking of objects at GEO, GTO, HEO and LEO. Currently ISON cooperates with 43 observation facilities of various affiliations with 100 telescopes in 17 countries. Six telescope subsets have been completed to the date, ISON encompasses five groups of telescopes and three scheduling centers. Obtained measurements are processed at the KIAM ballistic center to be used for scientific and applied goals, including collision risks analysis and space situation analysis. 20 millions measurements in 2.58 millions of tracklets for more 6740 objects have been collected by KIAM in 2016.
\end{abstract}

\section{RESUMEN}

La Red Científica Internacional Interagencias (ISON) constituye uno de los mayores sistemas para la observación de objetos espaciales. ISON proporciona monitoreado continuo de toda la región GEOestacionaria, observación regular de órbitas de objetos tipo Molniya, y seguimientos de objetos GEO, GTO, HEO y LEO. Actualmente, ISO coopera con 43 instalaciones observacionales con afiliaciones diversas, de un total de 100 telescopios pertenecientes a 17 países. Se han completado específicamente seis instalaciones, con ISON comprendiendo 5 grupos de telescopios y 3 centros de control. Las medidas obtenidas se procesan en el centro balístico KIAM usado para ciencia y otros objetivos, incluyendo análisis de riesgo de colisiones y de situación espacial. Desde 2016 se han obtenido 20 millones de medidas por medio de 2,58 millones de "tracklets" para un total de 6.740 objetos.

Key Words: space vehicles - telescopes

\section{INTRODUCTION}

The problem of studying the real population of space debris objects is extremely important. High Earth orbits are of great interest both for applied purposes of communication and navigation systems, and scientific researchers as well. Moreover Geostationary orbit (GEO) represents a limited natural resource which requires preservation for future use. There is no natural mechanism of self-cleaning here,

\footnotetext{
${ }^{1}$ Keldysh Institute of Applied Mathematics, Russian Academy of Sciences, Moscow, Russia (im62@mail.ru).

${ }^{2}$ Real Instituto y Observatorio de la Armada, San Fernando, Cadiz, Spain.

${ }^{3}$ Group of Astrodynamics for the Use of Space Systems, Rome, Italy.

${ }^{4}$ Sapienza University of Rome, Rome, Italy.

${ }^{5}$ Astronomical Institute, University of Bern, Bern, Switzerland.

${ }^{6}$ Ulugh Beg Astronomical Institute, Uzbek Academy of Sciences, Tashkent, Uzbekistan.

${ }^{7}$ Main Astronomical Observatory, National Academy of Sciences of Ukraine, Kiev, Ukraine.

${ }^{8}$ Universidad Autónoma Juan Misael Saracho, Observatorio Astronómico Nacional, Tarija, Bolivia.
}

similar to the low Earth orbit (LEO) region (decay of space debris fragments in the Earth atmosphere). Most of the GEO objects will live eternally, moving along the orbit. Monitoring of this region is a very topical goal due to the significantly increased number of the catalogued space objects there, including works on providing for the safety of satellites and the development of the proper model of space debris population.

Therefore Russian Academy of Science (RAS) initiated the national scientific program of space debris studying and commissioned to the Keldysh Institute of Applied Mathematics (KIAM) to establish the Center on collection, processing and analysis of information on space debris (CCPAISD) of the RAS. KIAM started the works aimed to systematic research of a population of small-sized high altitude objects and their properties and initiated the International scientific optical network (ISON) project (Molotov et al. 2008) for space debris observations. 
ISON is started as open international voluntary project developed to be an independent open source of data about space objects for scientific analysis and spacecraft operators. CCPAISD at KIAM is responsible for scheduling ISON instruments and processing all obtained information, maintenance of the ISON master database on space objects, related events (launches, fragmentations, re-entries, etc.), measurement data and derived products (orbits, etc.). Based on large amount of daily updating data CCPAISD performs analysis of situation in GEO region and provides different products (conjunction assessment messages, sets of raw measurements associated to given objects, orbital data/ephemerides, etc.) to customers which include Roscosmos, Russian GEO satellite operators and GEO satellite production enterprises, and also develops the model of space debris population at high Earth orbits (Usovik et al. 2017).

\section{INTERNATIONAL SCIENTIFIC OPTICAL NETWORK MILESTONES}

ISON started routine observations of the GEO objects in 2005 with support of the International Association for the promotion of co-operation with scientists from the New Independent States of the former Soviet Union and the Russian Ministry of education and science grants. On first stage of the project, space objects were tracked with existing obsolete telescopes that were inappropriate for this goal: small field of view (FOV), not-automated mount, not precise time keeping, etc. Observatory staff usually lacked the necessary experience and used various types of software for CCD image processing. And arranged telescope network modernization (purchasing the series of modern CCD-cameras) did not cause the expected effect on measurement rate. Therefore, it was solved to develop own telescopes, mounts, software etc. dedicated for space debris observations (Molotov et al. 2008) taking the focus on survey observations with usage of small inexpensive telescopes having large and very large FOV (Molotov et al. 2009). 12 series of telescopes (see characteristics in Table 1) and 5 series of WS mount with different weigh capacity, few set of domes and pavilions were elaborated, lot of individual solutions for automation of the mounts and optical scheme improvement of legacy telescopes that are working with ISON. More than 80 optical telescopes ranging apertures from $80 \mathrm{~cm}$ to $12.5 \mathrm{~cm}$ were produced and installed in observatories and also 12 obsolete telescopes of the $0.6-0.8 \mathrm{~m}$ class were refurbished. 40 mounts of WS-180-WS-500 were produced to complete new telescopes (small telescopes were installed on standard mount EQ6Pro and AZEQ). More than 100 CCD cameras of Finger Lakes Instrumentation were purchased (ML09000, ML16803, ML11002M, PL4301E, PL4240).

The standard time keeping hardware on base of the Trimble resolution T GPS receiver board for precise determination of CCD frame capture moment, utilizing the trigger mode of the CCD shutter was elaborated. About 100 time keeping devices were produced to date. It was also created a standard set of software modules for controlling of all telescope devices under a common platform - GPS receiver (AccuTime module), CCD camera (CameraControl module), telescope mount (CHAOS module), Apex II software package for astrometric and photometric reduction of the CCD frames (Kouprianov et al. 2013). All software components can interact with each other. Apex II provides calibration of CCD frames, detection of space objects, differential astrometry and photometry using stellar catalogs, identification of detected space objects using cata$\log$ of orbits and checking of the internal accuracy of measurements. This software set is used for space debris observation practically at all ISON observatories.

In last years a new integrated telescope control system and data acquisition software package FORTE5 (Facility for Operating Robotic Telescope Equipment) was developed (Kouprianov \& Molotov 2017).

The status of tens former Soviet Union observatories was checked and in addition, KIAM expeditions visited Argentina, Brazil, Bolivia, Bulgaria, Italy, Mexico, Mongolia and Venezuela to investigate the existing and potential observation point and collect an information on promising places for future installation of new ISON telescopes. Moreover a real international cooperation in space monitoring was arranged with many scientific institutions of many countries, starting in first turn with AIUB team in Switzerland (Zimmerwald observatory), TFRM project in Spain (Barcelona observatory), GAUSS team in Italy (Castelgrande observatory), Mexican universities (AUS in Sinaloa and UANL in Nuevo León), RCAG team in Mongolia (Khuraltogot observatory), etc.

For new small telescopes with FOV of 4 degree, the strategy of complete-twice surveys of Geostationary region in strip width of 18 degree with detection of all objects above a given magnitude was developed. Then following the development of telescopes with FOV of 7 degree, the method of extended GEO surveys (with up to 10 time passing of visible 
TABLE 1

CHARACTERISTICS OF TELESCOPES ELABORATED UNDER ISON PROJECT

\begin{tabular}{lllrrr}
\hline Name & Producer & $\begin{array}{l}\text { Optical } \\
\text { scheme }\end{array}$ & $\begin{array}{r}\text { Aperture/ } \\
\text { focus, mm }\end{array}$ & FOV, degree & CCD, mm \\
\hline VT-53e & Terebizh/Borisov & multiple lens & $125 / 204$ & 10 & 36 \\
VT-78a & Terebizh/Borisov & Schenker & $192 / 296$ & 7 & 36 \\
TGSH-200 & Yudin/Sankovicn & Hamilton & $200 / 307$ & 7 & 36 \\
SRT-220 & Terebizh/Borisov & Slevogt Richter & $220 / 507$ & 4 & 36 \\
ORI-22 & Terebizh/Borisov & Hamilton-Newton & $220 / 510$ & 4 & 36 \\
GAS-250 & Yudin/Sankovicn & Hamilton-Newton & $250 / 735$ & 2,85 & 36 \\
ORI-25 & Terebizh/Borisov & Hamilton-Newton & $250 / 625$ & 3,35 & 36 \\
SANTEL-400A & Yudin/Sankovicn & Hamilton-Newton & $400 / 1200$ & 1,75 & 36 \\
ORI-40 & Terebizh/Borisov & Hamilton-Newton & $400 / 920$ & 2,25 & 36 \\
ORI-50 & Terebizh/Borisov & Hamilton & $500 / 1160$ & 2,5 & 50 \\
ORI-50ML & Terebizh/Borisov & Hamilton & $500 / 970$ & 2 & 36 \\
SANTEL-650A & Yudin/Sankovicn & Hamilton & $650 / 1300$ & 2,2 & 50 \\
\hline
\end{tabular}

GEO region) for massive improving of orbits precision (thank to extra long measuring arcs) was designed. For middle class telescopes with FOV of 2 degree the strategy of local GEO survey to detect new faint fragments was developed.

Training courses were arranged for all teams of ISON observatories. Also annual workshops are regularly arranged for the common lectures of the ISON group representatives and the exchange of experience between the ISON observatory teams.

Above mentioned works allowed to KIAM quickly establish lot of new observation points to cover Geostationary orbit in the whole that allowed to maintain full catalogue of the GEO bright objects.

Since 2006 KIAM\&ISON are involved into the Roscosmos project "Automated System for Prediction and Warning on the hazardous situations in the near-Earth space" (ASPOS OKP). KIAM is responsible for conjunction analysis and notification at high Earth orbits and created the dedicated service for the daily operations. ISON is a main source of measuring data for this goal. First trial operations of ASPOS OKP system clearly displayed that existing data stream is not enough for quality collision risk analysis in high orbits. Therefore KIAM proposed to create a Roscosmos subnetwork of dedicated observation facilities (Molotov et al. 2014) to increase the volume of measurements and orbit determination precision, and in addition to the manufacturing of telescopes for two industrial companies interested in space monitoring.
The deployment of Roscosmos observatories that were produced based on the ISON experience was finished in 2015. Four mini-observatories EOP1 with 3 telescopes each: $40 \mathrm{~cm}$ (ORI-40 and SANTEL-400A), $25 \mathrm{~cm}$ (ORI-25 and GAS-250) and double $19.2 \mathrm{~cm}$ apertures (VT-78a) were installed in Kislovodsk (North Caucasus), Buyrakan (Armenia) and Nauchniy-3 (Crimea). Two miniobservatories EOP-2 with 3 telescopes in each: $65 \mathrm{~cm}$ (SANTEL-650A), $40 \mathrm{~cm}$ (ORI-40), 4x19.2 cm (VT78a) apertures were installed in Kislovodsk and Blagoveschensk. Three separate telescopes-OES$65(65 \mathrm{~cm}$ SANTEL-650A), OES-50 $(50 \mathrm{~cm})$ and OES-25 (25 cm ORI-25) were installed in Ussuriysk, Kislovodsk and Abrau-Durso.

Since 2016 new subset for activities with private commercial companies started the development under supervising of Small innovation enterprise (SME) "KIAM Ballistics-Service" Ltd. (Escobar et al. 2017) to separate the scientific and commercial aspects of the ISON activities.

\section{ISON CURRENT STATUS}

ISON project started as an open international voluntary project on self-financing basis represents now an interagency association that includes the 4 main segments with own scheduling centers and independent sources of the financing - scientific cooperation, Roscosmos ASPOS OKP subsystem, industry organizations collaboration and subset for commercial activities. In the whole, KIAM database receives 


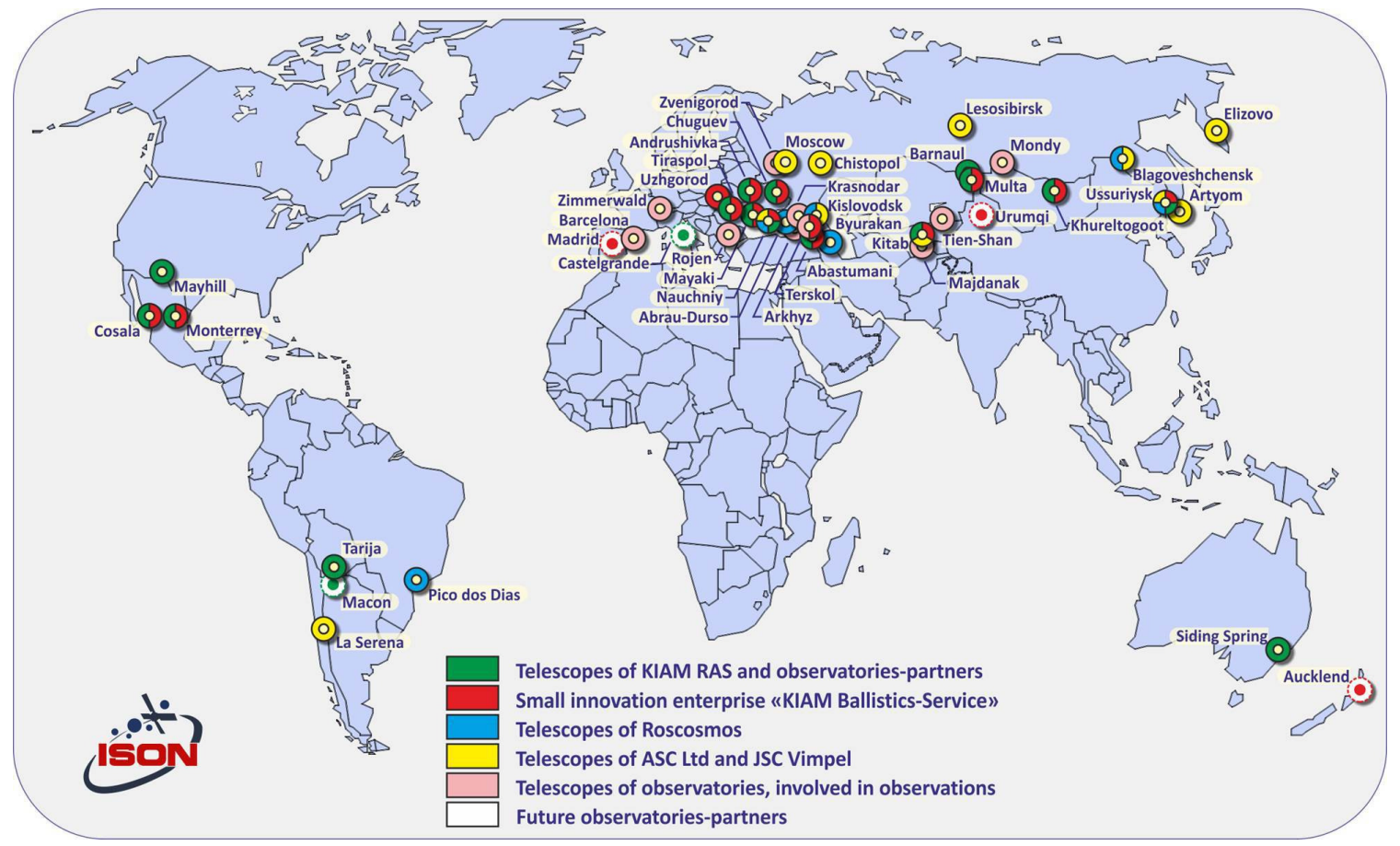

Fig. 1. Map of observatories that send the data to KIAM.

the measurements from 100 telescopes situated in 42 observation facilities of various affiliations in 17 countries (see Figure 1).

25 telescopes of KIAM international cooperation are applied for scientific researches (where each observatory is working on self-financing basis) represent the core of the ISON project. 25 telescopes of Roscosmos cooperation are used to support the conjunction analysis are used for conjunction analysis. 22 telescopes of cooperation of the Vimpel Corporation are working in interests of space situation analysis. 28 telescopes of SME "KIAM Ballistics-Service" Ltd are releasing for usage in commercial aspects (for customer requests service).

These telescopes carry out 5 types of observations: (1) standard GEO survey with $22-25 \mathrm{~cm}$ telescopes having FOV 3.5-4.4 degree, (2) extended GEO survey with 18-19.2 cm telescopes having FOV of 7-8 degree, (3) local (deep) GEO survey with 50$75 \mathrm{~cm}$ telescopes having FOV of 2-2.2 degree, (4) tracking of bright (brighter than apparent magnitude of 15.5) GEO and HEO objects telescopes of $25 \mathrm{~cm}$ aperture, (5) tracking of the faint (fainter than apparent magnitude of 15.5) space debris at GEO and GTO with $40-80 \mathrm{~cm}$ aperture telescopes. Additionally the tracking observations of LEO objects with $12.5-25 \mathrm{~cm}$ aperture telescopes and survey of HEO objects with $18-\mathrm{cm}$ telescope can be adjusted. Survey telescopes provide a main stream of the mea- surements for the catalogue maintenance and the new object detections. New bright objects then are tracked with telescopes operating in mode (4), new faint objects - with telescope operating in mode (5). The GEO surveys provide also much detection of the HEO/GTO objects. These objects then are tracked in mode (4).

\section{RESULTS OBTAINED}

Using ISON data, KIAM carries out the researches in the following fields: (i) estimation of real population of space debris at high geocentric orbits, (ii) determination of physical properties of discovered space debris objects, (iii) determination of probable sources of newly discovering space debris fragments, (iv) verification of existing evolution models of space debris distribution, (v) high orbit space debris risk assessment, (vi) improvement of technologies of studying of space debris population using optical instruments, (vi) improvement of motion models for space debris objects with complex physical properties.

The number of the measurements that is producing by the ISON is steadily growing each year (see Figure 2): 20.048 millions measurements in 2.58 millions of tracklets for 6740 objects at high orbits are collected by CCPAISD in 2017. Data on 2863 objects are obtained by ISON only (there is not TLE information) and orbits of 2080 these objects are reg- 


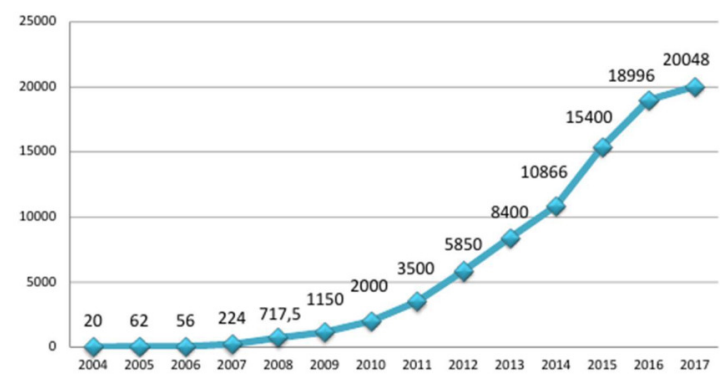

Fig. 2. Number of measurements (in thousands) collected by ISON annually for the 2004 - 2017 period.

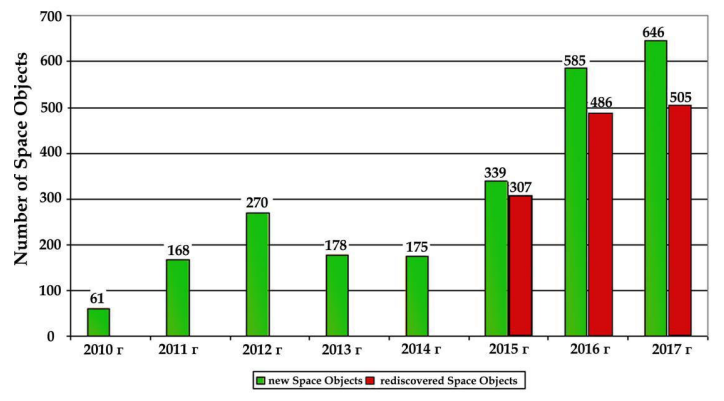

Fig. 3. Annual statistics of discovered and rediscovered by ISON objects.

ularly updated. Orbits of 5080 objects (2447 GEO, 2984 HEO and 402 MEO) are regularly updated in CCPAISD.

Figure 3 shows that in 2017646 new objects were discovered and 505 objects were rediscovered. The distribution of cataloged GEO objects as a function of the apparent magnitude is shown on Figure 4 . Figure 5 demonstrates the distribution of cataloged 2080 high orbit objects without TLE information as a function of area-to-mass ratio (AMR). At least half of this population is represented by objects with a high (more than $1 \mathrm{~m}^{2} / \mathrm{kg}$ ) area-to-mass ratio (HAMR).

So far, the number of discoveries of relatively bright GEO debris objects (brighter than 16-17 star magnitudes) continues to grow in spite of already meticulous surveys of all GEO ring, and at least half of this population is composed of HAMR-objects. This may demonstrate that there is some source of permanent generation of new GEO objects. Agapov et al. (2017) investigated possible reasons of these HAMR-objects origin. It was shown that the formation of HAMR-objects can be caused by processes leading, apparently, to degradation and destruction of the outer surface materials of the structural elements of the spacecraft and the launch vehicle upper stages.

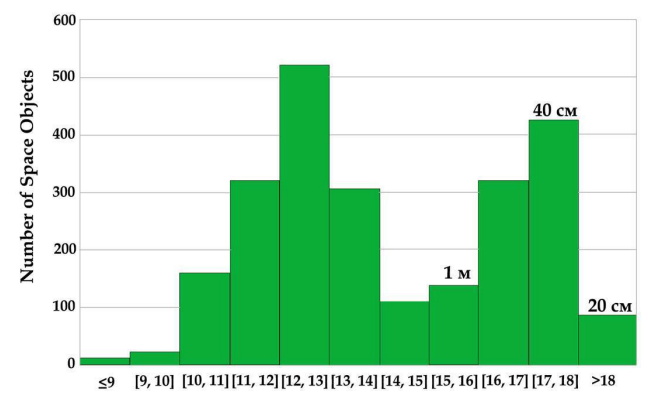

Fig. 4. The distribution of cataloged GEO objects by the value of the standard apparent magnitude.

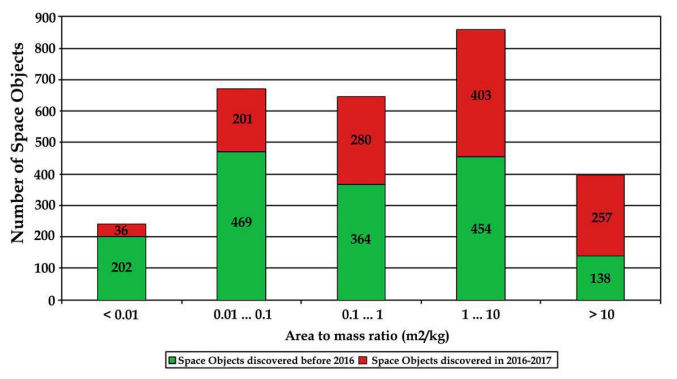

Fig. 5. Distribution of 2080 high orbit objects cataloged without TLE information by the value of AMR.

Many of newly discovered GEO space debris are crossing or permanently staying in the GEO protected region and increase threat to operational spacecrafts. Moreover, HAMR-objects can be formed directly in the area of the location of functioning spacecraft in GEO as well as HAMR-objects occur in graveyard orbits located above the GEO.

It is expected that thousands more of GEO space debris exist in the GEO region.

\section{CONCLUSIONS}

ISON project is continuing the development and quantity of instruments involved in space debris observation, the number of telescopes is already 100 in 42 observation sites. ISON is the rare example of fruitful international scientific collaboration in field of near-Earth orbits monitoring. And negotiations are carried out about collaboration of the KIAM scientific cooperation with "Open Universe" - new initiative of the United Nations Office for Outer Space Affairs.

ISON is split on 3 segments now (KIAM, Roscosmos and Vimpel) with own scheduling center and sources of finances. New "commercial" segment of 28 telescopes is forming and establishing the cooperation with the GMV Innovating Solution company. 
12 years of the ISON monitoring of GEO region is not resulting the discovering of all objects above given brightness. 1231 new space objects were detected in last two year, and about $50 \%$ are HAMRobjects. Next step in investigation of GEO population region will be connected with putting in operation of telescopes having larger aperture and larger FOV.

It is planned to improve the HEO objects observations by elaboration of new scheduling software and producing the new optical telescopes - "barrier" (observations in perigee to improve the orbit accuracy) and "panoramic" surveys (for detection of new objects).

\section{REFERENCES}

Agapov, V. 2017, Proc. 68th International Astronautical

$$
\text { Congress, A6.1.8x41827 }
$$

Escobar, D., et al. 2017, ESA/ESOC, Proc. 7th European Conference on Space Debris, id. 414

Kouprianov, V. 2013, ESASP 723, Proc. 6th European Conference on Space Debris, ed. L. Ouwehand, 21

Kouprianov, V. \& Molotov, I. 2017, ESA/ESOC, Proc. 7th European Conference on Space Debris, id. 475

Molotov, I., et al. 2008, AdSpR, 41, 1022

Molotov, I., et al. 2008, Proc. 59th International Astronautical Congress, IAC-08-A6.1.09, (New York, NY: Curran Associates), 2216

Molotov, I., et al. 2009, Proc. 60th International Astronautical Congress, IAC-09.A6.1.8, (New York, NY: Curran Associates), 1804

Molotov I., et al. 2014, Proc. 65th International Astronautical Congress, IAC-14.A6.1.4, (New York, NY: Curran Associates), 1177

Usovik, I. Stepanov, D., \& Stepanyats, V. 2017, Proc. 7th European Conference on Space Debris, id 480 